\title{
EXTENSIONS OF MEASURES AND THE VON NEUMANN SELECTION THEOREM ${ }^{1}$
}

\author{
ARTHUR LUBIN
}

\begin{abstract}
Let $\left(X, B_{X}\right)$ be a Blackwell space, where $B_{X}$ is the $\sigma$-algebra of Borel sets. Then if $\sigma$ is a finite measure defined on a countably generated sub- $\sigma$-algebra $B \subset B_{X}, \sigma$ can be extended to a Borel measure $\tau$. Equivalently, if $X$ and $Y$ are Blackwell and $f: X \rightarrow Y$ is Borel, and $\mu$ is a Borel measure carried on $f(X) \subset Y$, then there exists a Borel measure $\tau$ on $X$ with $\tau^{f}=\sigma$, where $\tau^{f}(E)=\tau\left(f^{-1}(E)\right)$. We characterize $\left\{\tau \mid \tau^{f}=\sigma\right\}$ if $f$ is semischlicht.
\end{abstract}

Let $B_{X}$ denote the Borel sets of a topological space $X$. We consider the following measure extension (or equivalently restriction) problem: given a measure (we will always mean finite measure) $\sigma$ defined on a $\sigma$-algebra $B \subset B_{X}$, can $\sigma$ be extended to all of $B_{X}$, i.e., does there exist a Borel measure $\tau$ such that $\tau(E)=\sigma(E)$ for all $E \in B$ ? It is well known (see [1, p. 71], for details) that if $B_{1}$ and $B_{2}$ are $\sigma$-algebras, and $B_{2}$ is generated by $B_{1}$ and finitely many additional sets, then any measure on $B_{1}$ can be extended to $B_{2}$. The result is not known for countably generated extensions. We show below (Theorem 5) that if $X$ is a Blackwell space and $B$ is a countably generated sub- $\sigma$-algebra of $B_{X}$, then any measure on $B$ extends to $B_{X}$.

A Blackwell space is a measure space $\left(X, B_{X}\right)$, where $X$ is an analytic subset of a complete separable metric space (c.s.m.). A subset $A$ of a c.s.m. is analytic iff $A$ is the continuous image of a c.s.m. We note that the analytic sets form a proper subset of $U_{X}$, the set of absolutely measurable subsets of $X$, where $E \in U_{X}$ iff $E$ is $\bar{\mu}$-measurable for all finite Borel measures $\mu$, where " $\vec{\mu}$ " denotes the completion of $\mu$, i.e., given $\mu$, there exist $E_{1}, E_{2} \in B_{X}$ such that $E_{1} \subset E \subset E_{2}$ and $\mu\left(E_{2}-E_{1}\right)=0$. A function $g$ is said to be absolutely measurable if $g^{-1}(V) \in U_{X}$ for all open $V$. Details may be found in [3], [4], or [5]. We note that if $X \subset S, X$ analytic, $S$ a c.s.m., then $B_{X}=\left\{E \cap X \mid E \in B_{S}\right\}$, so elements of $B_{X}$ are topologically analytic, and not necessarily Borel in $S$.

We begin by considering a special class of sub- $\sigma$-algebras of $B_{X}$. Let $f: X \rightarrow Y$ be Borel measurable, and let $B_{f}=\left\{f^{-1}(E) \mid E \in B_{X}\right\}$. Given a Borel

Presented to the Society, April 20, 1973; received by the editors January 30, 1973 and, in revised form, June 4, 1973.

AMS (MOS) subject classifications (1970). Primary 28A05, 28A60, 28-00.

${ }^{1}$ Partially supported by NSF GP-38265.

(C) American Mathematical Society 1974 
measure $\tau$ on $X$, let $\tau^{f}$ be the Borel measure in $Y$ defined by $\tau^{f}(E)=$ $\tau\left(f^{-1}(E)\right)$. It follows from a well known isomorphism theorem (see [7, p. 270]) that if $\tau$ is a positive Borel measure on a c.s.m. $X$, and $\mu$ is a positive Borel measure on a c.s.m. $Y$ with $\mu(Y)=\tau(X)$ and $\tau(\{x\})=0$ for all $x \in X$, then there exists a Borel function $f: X \rightarrow Y$ with $\tau^{f}=\mu$. The following shows that a dual of this is equivalent to our extension problem.

Proposition 1. Let $f: X \rightarrow Y$ be Borel measurable. Then every measure on $B_{f}$ is the restriction of a Borel measure iff for every $\mu$ defined on $B_{Y}$ with $\bar{\mu}\left(f(X)^{c}\right)=0$ (i.e., $\mu$ is carried on $f(X)$ ), there exists $\tau$ on $B_{X}$ with $\tau^{f}=\mu$.

Proof. Assume that all Borel $\mu$ carried on $f(X) \subset Y$ are of the form $\mu=\tau^{f}$, and let $\sigma$ be a measure on $B_{f} \subset B_{X}$. Define $\mu$ on $B_{Y}$ by $\mu(E)=$ $\sigma\left(f^{-1}(E)\right), E \subset Y$. By assumption, there exists a Borel measure $\tau$ with $\tau^{f}=\mu$. Thus, $\tau\left(f^{-1}(E)\right)=\mu(E)=\sigma\left(f^{-1}(E)\right)$, so $\tau$ extends $\sigma$.

Conversely, suppose the extension property holds. Then given a Borel measure $\mu$ carried on $f(X) \subset Y$, define $\sigma$ on $B_{f}$ by $\sigma\left(f^{-1}(E)\right)=\mu(E)$. (Note $\sigma$ is well-defined since $\mu$ is carried on $f(X)$.) By assumption, $\sigma$ is the restriction of some $\tau$ defined on $B_{X}$, and clearly $\tau^{f}=\mu$.

The proof of our main result relies on the following "selection" theorem of von Neumann.

THEOREM 2 [6]. Let $A$ be an analytic subset of a c.s.m. S, and let $F$ be a continuous real-valued function on $A$. Then there exists an absolutely measurable $G: F(A) \rightarrow A$ such that $F \circ G$ is the identity on $F(A)$.

Corollary 3. If $X$ and $Y$ are Blackwell and $F: X \rightarrow Y$ is Borel, there exists an absolutely measurable $G: Y \rightarrow X$ such that $F \circ G$ is the identity on $F(X)$.

Proof. Since $Y$ is Blackwell, we may assume $Y \subseteq \boldsymbol{R}$, the real line. Let $(F \times I):(X \times Y) \rightarrow(Y \times Y)$ be defined by $(F \times I)((x, y))=(F(x), y)$, and let $P_{j}$ be projection on the $j$ th coordinate, $j=1,2$. Then

$$
A=\{(x, F(x)) \mid x \in X\}=(F \times I)^{-1}(\{(y, y)\})
$$

is Borel in $X \times Y$, and is thus an analytic subset of a c.s.m. Since $P_{2}: A \rightarrow Y$ is continuous, there exists an absolutely measurable $g: P_{2}(A) \rightarrow A$ with $P_{2} \circ g$ the identity on $P_{2}(A)=F(X)$. Then $G=P_{1} \circ g$ satisfies the theorem.

REMARK 4. (i) If $f: X \rightarrow Y$ is Borel and $B_{Y}$ is countably generated, then $B_{f}$ is countably generated.

(ii) If $X$ is Blackwell and $B \subset B_{X}$ is countably generated, then there exists a Borel $f: X \rightarrow \boldsymbol{R}$ with $B=B_{f}$. (This was pointed out to me by the referee.)

Proof. (i) follows immediately. 
Suppose $\left\{E_{n}\right\}$ generates the $\sigma$-algebra $B$. Consider $f(x)=\sum_{n} 3^{-n} \chi_{E_{n}(x)}$. Then since $B$ and $B_{f}$ clearly have the same atoms and $X$ is Blackwell, it follows that $B=B_{f}$ (see [5, p. 38]).

THeOREM 5. Let $X$ be Blackwell and let $\sigma$ be a finite measure on a countably generated sub- $\sigma$-algebra $B$. Then $\sigma$ has an extension $\tau$ to the full $\sigma$-algebra $B_{X}$.

We first prove the following

Lemma. If $f: X \rightarrow Y$ is absolutely measurable and $S \subset Y$ is absolutely measurable, then $f^{-1}(S) \subset X$ is absolutely measurable. In particular, a composition of absolutely measurable functions is absolutely measurable.

Proof. Given any $\mu$ on $B_{X}$, consider $\nu=(\bar{\mu})^{f}$ defined on $B_{Y}$. Since $S$ is absolutely measurable, there exist $S_{1}, S_{2} \in B_{Y}$ with $S_{1} \subset S \subset S_{2}$, $v\left(S_{2}-S_{1}\right)=0$. Let $T_{i}=f^{-1}\left(S_{i}\right)$. Then $T_{1} \subset f^{-1}(S) \subset T_{2}, T_{1}, T_{2}$ are absolutely measurable, and $\bar{\mu}\left(T_{2}-T_{1}\right)=\bar{\mu}\left(f^{-1}\left(S_{2}-S_{1}\right)\right)=v\left(S_{2}-S_{1}\right)=0$. Thus, $f^{-1}(S)$ is $\bar{\mu}$-measurable.

Proof of Theorem 5. Given $\sigma$ on $B$, we have $B=B_{f}$ where $f: X \rightarrow R$ is Borel. Then there exists an absolutely measurable $g: f(X)=Y \rightarrow X$ with $f \circ g=i_{Y}$. Define $\tau$ on $B_{X}$ by $\tau(S)=\bar{\sigma}\left((g \circ f)^{-1}(S)\right)$. (This makes sense, since $g \circ f$ is absolutely measurable.) Clearly, if $S \in B_{f}$, there exists $T \subset Y$ such that $S=f^{-1}(T)$ and $T \in B_{Y}$.

Then

$$
\begin{aligned}
\tau(S) & =\bar{\sigma}\left((g \circ f)^{-1}(S)\right)=\bar{\sigma}\left(f^{-1}\left(g^{-1}(S)\right)\right) \\
& =\bar{\sigma}\left(f^{-1}\left((f \circ g)^{-1}(T)\right)\right)=\bar{\sigma}\left(f^{-1}(T)\right) \\
& =\bar{\sigma}(S)=\sigma(S) .
\end{aligned}
$$

Thus, $\tau$ extends $\sigma$ to $B_{X}$.

Corollary 6. Let $f: X \rightarrow Y$ be Borel, where $X$ and $Y$ are Blackwell, and let $\mu$ be a Borel measure carried on $f(X) \subset Y$. Then there exists $\tau$ on $B_{X}$ with $\tau^{f}=\mu$.

The proof of the corollary is explicit in the proof of the theorem. We note that the $\tau$ obtained is of the form $\tau(E)=\mu\left(g^{-1}(E)\right)=\mu(f(E \cap S))$, where $S=g(Y) . S$ thus consists of one point "selected" from each preimage set $f^{-1}(\{y\})$. Hence, $\tau$ is carried on a 1-1 set, i.e., a section, of $f$.

If $f$ is $1-1$, then $\tau$ is clearly unique. Suppose that $f$ is semischlicht, i.e., that $f^{-1}(\{y\})$ is a countable set for all $y \in Y$. Then there is a countable collection of disjoint Borel sets $\left\{S_{n}\right\}$ such that $\bigcup S_{n}=X, f_{n}=\left.f\right|_{S_{n}}$ is 1-1, and $f\left(S_{n}\right)=f\left(X-\bigcup_{j<n} S_{j}\right)$. (See [2, p. 335].) Given a Borel measure $\sigma$ on $Y$, let $\tau_{n}$ be defined on $B_{X}$ by $\tau_{n}(E)=\sigma\left(f\left(E \cap S_{n}\right)\right)$. Then $\tau_{n}^{f_{n}}=\left.\sigma\right|_{f_{n}(X)}$. 
Choose $a_{n} \in L^{1}\left(\tau_{n}\right)$ such that $\sum_{n} a_{n}\left(f_{n}^{-1}(y)\right)=1$ a.e. [ $\left.\sigma\right]$, where

$$
a_{n}\left(f_{n}^{-1}(y)\right)=0 \quad \text { if } y \notin f_{n}\left(S_{n}\right),
$$

and the series is absolutely convergent. Let $\tau$ be defined on $B_{X}$ by $\tau(E)=\sum_{n} \int a_{n}(x) d \tau_{n}(x)$, i.e., $d \tau(x)=\sum_{n} a_{n}(x) d \tau_{n}(x)$ so $\tau$ is a "convex combination" of the measures $\tau_{n}$. Then it is easy to see that $\tau^{f}=\sigma$, and we see below that this characterizes $\left\{\tau \mid \tau^{f}=\sigma\right\}$.

Proposition 7. Let $X$ and $Y$ be Blackwell, and let $f: X \rightarrow Y$ be a semischlicht Borel map. Let $\sigma$ be a measure defined on $B_{Y}$, and suppose that $\left\{S_{n}\right\},\left\{f_{n}\right\}$ and $\left\{\tau_{n}\right\}$ are as above. Then there exists $\left\{a_{n}(x)\right\}$ such that $a_{n} \in$ $L^{1}\left(\tau_{n}\right), \sum_{n} a_{n}\left(f_{n}^{-1}(y)\right)=1$ a.e. $[\sigma]$, and $d \tau(x)=\sum_{n} a_{n}(x) d \tau_{n}(x)$.

Proof. Using the Hahn decomposition, we may assume that $\sigma$ and $\tau$ are positive. Then for $E \subset S_{n} \subset X$,

$$
\tau(E)=\tau\left(f_{n}^{-1}\left(f_{n}(E)\right)\right) \leqq \tau\left(f^{-1}\left(f_{n}(E)\right)\right)=\sigma\left(f_{n}(E)\right)=\tau_{n}(E),
$$

so by the Radon-Nikodym theorem, there exists $a_{n} \in L^{1}\left(\tau_{n}\right)$ with

$$
\tau(E)=\int_{E} a_{n}(x) d \tau_{n}(x)=\int_{f_{n}(E)} a_{n}\left(f_{n}^{-1}(y)\right) d \sigma(y) \quad \text { if } E \subset S_{n} .
$$

Thus, for $E \subset X$,

so

$$
\tau(E)=\sum_{n} \tau\left(E \cap S_{n}\right)=\int_{E} \sum a_{n}(x) d \tau_{n}(x),
$$

For $E \subset Y$,

$$
d \tau(x)=\sum_{n} a_{n}(x) d \tau_{n}(x) .
$$

Hence,

$$
\begin{aligned}
\sigma(E)=\tau\left(f^{-1}(E)\right) & =\sum \int_{f_{n}\left(f^{-1}(E) \cap S_{n}\right)} a_{n}\left(f_{n}^{-1}(y)\right) d \sigma(y) \\
& =\int_{E}\left(\sum_{n} a_{n}\left(f_{n}^{-1}(y)\right)\right) d \sigma(y) .
\end{aligned}
$$

$$
\sum_{n} a_{n}\left(f_{n}^{-1}(y)\right)=1 \text { a.e. }[\sigma] \text {. }
$$

We point out that if, in fact, $\tau(E)=\sigma(f(E \cap S))$ for $E \subset X$, where $\left.f\right|_{S}$ is $1-1$, then for each $n$, there exists $D_{n} \subset S_{n}$ such that $a_{n}=\chi_{D_{n}}$ and $S=\bigcup_{n} D_{n}$. Further, the following example shows that the assumption $f$ semischlicht cannot be dropped.

For $a \in[0,1]$ an irrational number, consider the unique decimal representation $a=0 . a_{1} a_{2} \cdots$, and let $f:[0,1] \rightarrow[0,1]$ be defined by $f\left(0 . a_{1} a_{2} a_{3} \cdots\right)=0 . a_{1} a_{3} a_{5} \cdots$. Then, after defining $f$ in an appropriate manner on the rational points, which we can ignore since we will be using 
nonatomic measures, $f$ maps $[0,1]$ onto $[0,1]$, and

$$
f^{-1}\left(0 . a_{1} a_{2} \cdots\right)=\left\{0 . a_{1} x_{1} a_{2} x_{2} \cdots \mid x_{i} \text { arbitrary }\right\} .
$$

Let $\Phi:[0,1] \rightarrow([0,1] \times[0,1])$ be defined by

$$
\Phi\left(0 . a_{1} a_{2} a_{3} \cdots\right)=\left(0 . a_{1} a_{3} a_{5} \cdots, 0 . a_{2} a_{4} a_{6} \cdots\right) .
$$

Let $m$ be Lebesgue measure in $[0,1]$. Let $\sigma$ be a singular nonatomic measure on $[0,1]$. We now define $\tau$ on $[0,1]$ by $\tau(E)=(\sigma \times m)(\Phi(E))$. We note that $\Phi(E)$ is measurable since $\Phi$ restricted to the irrationals is a homeomorphism.

Suppose $E=f^{-1}(B)$ for some Borel set $B \subset[0,1]$. Then Thus,

$$
E=\left\{0 . a_{1} x_{1} a_{2} x_{2} \cdots \mid 0 . a_{1} a_{2} \cdots \in B\right\} \text { and } \Phi(E)=B \times[0,1] .
$$

$$
\begin{aligned}
\tau(E) & =\tau\left(f^{-1}(B)\right)=(\sigma \times m)(\Phi(E)) \\
& =(\sigma \times m)(B \times[0,1])=\sigma(B) .
\end{aligned}
$$

Hence, $\tau^{f}=\sigma$, and it is easy to see that if $\left.f\right|_{S}$ is $1-1$, then $\tau(S)=0$, so $\tau$ cannot be written as a "combination" of measures supported on 1-1 sets of $f$.

Acknowledgment. The author is grateful to Professors P. R. Halmos, Edward Fisher, and William Moran for pointing out a reference to von Neumann's paper and thus simplifying an earlier version of this paper; and also to the referee for pointing out the notion of Blackwell spaces, and other helpful comments.

\section{BIBLIOGRAPHY}

1. P. R. Halmos, Measure theory, Van Nostrand, Princeton, N.J., 1950. MR 11, 504.

2. F. Hausdorff, Set theory, 2nd ed., Chelsea, New York, 1962. MR 25 \#4999.

3. K. Kuratowski, Topologie. Vol. I, 2nd ed., Monografie Mat., Tom 20, WarszawaWroclaw, 1948; English transl., New ed., rev. and aug., Academic Press, New York; PWN, Warsaw, 1966. MR 10, 389; 36 \#840.

4. N. Lusin, Leçons sur les ensembles analytiques, Hermann, Paris, 1930.

5. Paul A. Meyer, Probability and potentials, Blaisdell, Waltham, Mass., 1966. MR 34 \#5119.

6. J. von Neumann, On rings of operators. Reduction theory, Ann. of Math. (2) 50 (1949), 401-485. MR 10, 548.

7. H. L. Royden, Real analysis, Macmillan, New York; Collier-Macmillan, London, 1963. MR 27 \#1540.

Department of Mathematics, University of Wisconsin, Madison, Wisconsin 53706

Current address: Department of Mathematics, Northwestern University, Evanston, Illinois 60201 